\title{
Trends in Intervention Studies on Food and Nutrition Education in Japan Utilizing the School Lunch - A Systematic Review
}

\author{
Yoko Hosoyamada*1 and Kimiko Miyahara*2 \\ ${ }^{* 1}$ Department of Nutrition and Dietetics, College of Nutrition, Kanto Gakuin University \\ ${ }^{* 2}$ Department of Nutrition, Faculty of Health Care, Kiryu University
}

doi:10.5264/eiyogakuzashi.76.S50

\begin{abstract}
Purpose: A systematic review was undertaken for the purpose of determining trends in food and nutrition education utilizing school lunches following the enactment of the Shokuiku Basic Act in 2005.

Method: A search for academic papers published over the 12-year period from 2005 to 2016 was conducted using the database of the Japan Medical Abstracts Society as well as a manual search. The criteria for selection were as follows: Firstly, papers had to be published in reviewed academic journals. Secondly, papers had to be original articles. Thirdly, the subjects of the papers had to be Japanese elementary school or junior high school students. Fourthly, the contents had to relate to the school lunch program. Fifthly, the papers had to contain statistical analyses.

Results: A total of 165 papers were selected from the database search and 86 papers from the manual search. Eleven papers were ultimately chosen. The contents of these papers comprised food and nutrition education taught in the classroom (eight papers) and food and nutrition education that utilized school lunch times (three papers). The reports were published following the enactment of the Shokuiku Basic Act in 2005 and the School Lunch Act in 2008. Three papers were published in the period 2005 to 2010 and eight in the period 2011 to 2016.

Conclusion: In recent years there has been an increase in the number of reports relating to food and nutrition education using school lunches with the recommendation that this method of education becomes part of the curriculum.
\end{abstract}

Jpn. J. Nutr. Diet., Vol.76 Supplement 1 S50-S63

(2018)

Key words: school lunch, diet and nutrition teacher, food and nutrition education, team teaching, systematic review

\section{Introduction}

The Shokuiku Basic Act ${ }^{1)}$ was enacted in June 2005 for the purpose of cultivating a healthy mind and body among citizens over the course of their entire lives and nurturing a rich sense of humanity in response to lifestyle and social changes accompanying Japan's rapid economic growth and an increasingly diversified sense of values regarding individual eating habits and food. The preamble of the Shokuiku Basic Act emphasizes shokuiku targeted at children in particular; "Food, more than anything else, is important to ensure that children develop a rich sense of humanity and the strength to live." Moreover, Article 5 of this Act mentions the roles played by parents and school personnel in children's shokuiku, indicating that aggressive steps should be taken to promote shokuiku: "Persons engaged in the education and upbringing of children must have an adequate awareness of the importance of shokuiku and take the necessary steps to promote shokuiku among children." Article 20 discusses the proliferation of knowledge regarding the establishment of a guidance system, the implementation of a school lunch program that takes advantage of characteristics unique to the school or local community and the promotion of shokuiku among children through various learning experiences conducted as part of the educational curriculum. It also discusses the effects of under-eating or obesity on health and measures to be deployed to encourage healthy eating habits among children along with healthy mental and physical growth through the effective promotion of appealing food and nutrition education at schools, day care centers and other educational institutions. This indicates the considerable

Corresponding Author: Yoko Hosoyamada. College of Nutrition, Kanto Gakuin University, 1-50-1 Mutsuurahigasi, Kanazawa-ku, Yokohama 236-8503, Japan

Tel: +81-45-786-5625 E-mail: hoso@kanto-gakuin.ac.jp 
expectation placed on the school lunch program and on food and nutrition education.

In response to these trends, the School Lunch $\mathrm{Act}^{2)}$, following its revision in June 2008, stipulated the promotion of shokuiku through the school lunch program and enhanced educational elements of the school lunch program. According to the School Lunch $\mathrm{Act}^{2)}$, the school lunch program is positioned as part of school education. Article 1 of this law stipulates essential matters relating to the school lunch program and the implementation of food and nutrition education utilizing the school lunch program by indicating that it contributes to the healthy mental and physical development of elementary school or junior high school students and fulfills an important role in terms of fostering correct knowledge and the ability to make proper decisions relating to food by these students. In addition, the promotion of food and nutrition education at schools was once again stipulated to enhance the proliferation of the school lunch program and the promotion of shokuiku at schools. The role of a diet and nutrition teacher was legislated to develop school lunch menus as teaching materials for food and nutrition education. This places considerable importance on the school lunch menu as a teaching aid for the formation of desirable eating habits targeted at school children during their stage of growth and development.

There have been numerous reports, including those at schools in Japan, on the practical implementation of food and nutrition education, its management and the coordination of teaching materials. Although there have recently been reports on trends in intervention studies relating to school-based food and nutrition education ${ }^{3)}$ as well as systematic reviews relating to educational programs at cooking schools focusing on food and nutrition education ${ }^{4)}$ in various foreign countries, there have hardly been any reports of systematic reviews comprising a detailed organization and analysis of intervention contents and methods regarding the utilization of food and nutrition education and teaching materials during school lunches in Japan. On the basis thereof, the purpose of this study is to determine trends in food and nutrition education utilizing the school lunch following the enactment of the Shokuiku Basic Act in 2005 by focusing on intervention research on this topic in Japan and organizing that research according to subjects, intervention contents and major evaluation criteria.

\section{Methods}

\section{Extraction of papers}

Papers were extracted from a database as well as a manual search.

1) Standardization of research themes

Prior to gathering papers, the PICO method (Population, Intervention, Comparator, Outcome) was used to set the research theme for the systematic review with respect to the subjects of the study, intervention method, comparative measurement method and outcome ${ }^{5,6)}$. With regards to this study, $\mathrm{P}=$ elementary school or junior high school students, I = interventions relating to food and nutrition education correlated with school lunch times, teaching materials, moral education, foreign language activities, time allotted for integrated studies and special activities utilizing school lunches, $\mathrm{O}=$ what types of effects are observed and $\mathrm{C}=$ before and after intervention or between an intervention group and control group.

2) Selection of target papers

The criteria for selecting papers was as follows: Firstly, papers had to be extracted from reviewed academic journals excluding bulletins and proceedings. Secondly, papers had to be original, concise and practical (excluding proceedings, case reports and commentaries). Thirdly, the subjects of the papers had to be Japanese elementary school or junior high school students. Fourthly, the contents had to be related to school lunches. Fifthly, the papers had to contain statistical analyses. Finally, the contents had to have been published in the period from 2005 to 2016.

Primary screening consisted of carefully examining titles and abstracts and excluding those papers that did not match the selection criteria. Next, secondary screening was conducted on those papers selected in primary screening. Secondary screening consisted of obtaining and carefully reading the papers and choosing target papers based on the selection criteria. The target papers were selected by two persons, and in cases in which the decisions differed between these two persons, final selection was decided through discussions.

3) Database search

The database search was conducted on 12 May 2017 
Table 1 Search Words and Search Format for Papers Examining Food and Nutrition Education at School Lunches in Japan Using the Japan Medical Abstracts Society Database

\begin{tabular}{ll}
\hline \multicolumn{1}{c}{ Group } & \multicolumn{1}{c}{ Search Term } \\
\hline 1. Subjects & (School/TH or School/AL) \\
\hline 2. School Lunch & (School lunch/TH or School lunch/AL, (School lunch/TH or School lunch/AL) \\
\hline 3. Food/Nutrition Education & $\begin{array}{l}\text { (Food education/TH or Food education/AL), Food education/AL, (Nutrition education/TH or } \\
\text { Nutrition education AL), (Eating behavior/TH or Eating behavior/AL), Food education/AL or Eat- } \\
\text { ing attitude/AL or Food knowledge/AL or School lunch guidance/AL }\end{array}$ \\
\hline
\end{tabular}

Limit conditions: 2005 2016, people, children, excluding case studies, case reports and proceedings.

Search format: Search terms of each group connected with OR, each group linked with AND, searches made according to limit conditions.

TH: Thesaurus, AL: Partial match search targeted at all data.

Search date: May 12, 2017

using Web Version 5 of the Japan Medical Abstracts Society (JMAS) database. The search terms and search format are shown in Table 1 . The search terms were obtained by dividing key words relating to this study into three groups based on PICO, connecting the terms in the groups with OR, linking groups with AND, and setting limit conditions.

4) Manual search

The manual search was conducted by referencing previous studies ${ }^{7,8)}$. Fifteen journals containing articles on research relating to school education, school lunches, food and nutrition education or nutrition education were targeted. They were the Japanese Journal of Nutrition and Dietetics, Journal of the Family Education Research Center, Japanese Journal of School Health, Journal of the Food Hygienic Society of Japan, Journal of Child Health, Journal of the Japan Dietetic Association, Journal of Nutritional Science and Vitaminology, Journal of Home Economics of Japan, Japanese Journal of Home Economics Education, Bulletin of Japanese Curriculum Research and Development, Japanese Journal of Health Education and Promotion, Japanese Journal of Public Health, Journal of Japanese Society of Shokuiku, Journal for the Integrated Study of Dietary Habits and Journal of Japan Society for the Study of Obesity.

This study contains food and nutrition education in the search terms. Since the JMAS database used for the search mainly contains research relating to medicine, dentistry, pharmacology, nursing, veterinary science and related fields ${ }^{9)}$ and frequently rejects subject matter relating to food science ${ }^{10)}$, it was thought that there would be limitations regarding coverage of papers relating to this study when using this database alone. The extraction of papers by manual search employed the same search terms and conditions as those used in the database search. In extracting papers, tables of contents were examined from index numbers indicated in academic society journals and websites in the database. Since some journals do not allow access to full abstracts only those that did were examined along with their titles.

\section{Production of evidence tables}

Evidence tables were produced and organized according to purpose, study subjects, intervention contents, evaluation times, evaluation methods and major results.

\section{Results}

\section{Extraction of papers}

The results of the article search are shown in Figure 1. One hundred and sixty five papers were extracted from the JMAS database from which 28 papers were selected based on a careful examination of their titles and abstracts. Subsequently these papers were carefully read in their entirety ultimately resulting in the selection of five papers. During the manual search, 86 papers were selected from 15 academic journals through a careful examination of titles and abstracts in the same manner as the database search, after which the entire contents of the papers were read thoroughly, ultimately resulting in the selection of six papers. Accordingly, a total of 11 papers were selected from a combination of the database and manual search.

The ultimately selected papers were classified into two evidence tables according to the type of intervention, and were organized for purpose, methods and major results. The contents of the selected papers consisted of eight papers describing food and nutrition education provided in the classroom ${ }^{11 \sim 18)}$ (Table 2) and three papers describing 


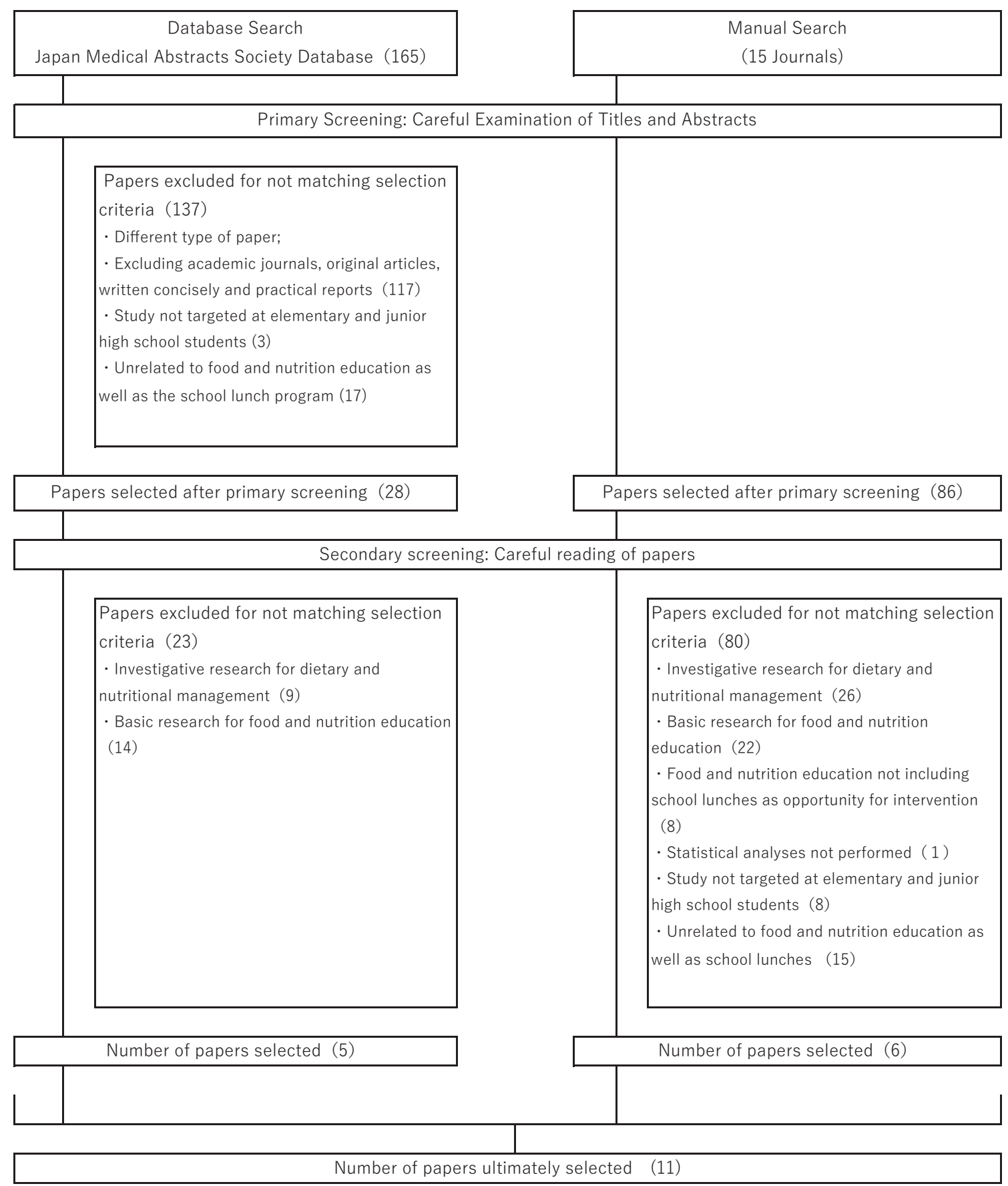

Figure 1 Flow Chart of Selection of Papers

Figures in parentheses ( ) indicate the number of papers.

food and nutrition education provided during lunch times $^{19 \sim 21)}$ (Table 3). School courses offered in the classroom refer to all subjects, moral education, foreign language activities, integrated studies and special activities as indicated in The Course of Study ${ }^{22)}$.

\section{Years of publication and number of papers}

The years of publication and number of papers ultimately selected consisted of two papers published in $2008^{11,12)}$, one in $2009^{19)}$, one in $2011^{13)}$, one in $2012^{14)}$, one in $2013^{15)}$, four in $2015^{16 \sim 18,20)}$ and one in $2016^{21)}$. All reports were published after 2008 following the revision of the School Lunch Act in that year - three in the period 2008 to 2010 and eight from 2011 to 2016 .

\section{Study subjects}

The subjects targeted in these papers consisted of elementary school students ${ }^{11,13 \sim 17,19,20)}$ (eight papers), elementary school students and junior high school students $^{12)}$ (one paper), elementary school students and their 
parents $^{18)}$ (one paper) and elementary school students and teachers ${ }^{21)}$ (one paper). The school year having the largest number of reports was sixth graders ${ }^{11 \sim 13,16,17,19)}$ (six papers), with three papers targeted at fourth graders $^{15,18,20)}$, two papers targeted at fifth graders ${ }^{12,13)}$ and one paper targeted at second graders ${ }^{14)}$. In addition, two reports targeted all elementary school grades ${ }^{18,21)}$ and one report targeted first year junior high school students $^{12)}$.

\section{Major study purpose}

The purpose of the intervention research described in the papers consisted of ten papers targeted at behavioral changes attributable to the effectiveness of food and nutrition education programs. Six of these papers focused on the amount of leftovers of school lunches or improvement in the amount of food consumed ${ }^{12,14,15,19 \sim 21)}$ and four papers targeted combinations of foods and improvement of meal contents ${ }^{11,13,16,18)}$. In addition to behavioral changes, these papers also targeted improvement in attitude towards food such as greater enjoyment from school lunches ${ }^{12,18,20)}$, improvement in understanding and awareness of food such as distinguishing between staple foods, main dishes and side dishes ${ }^{13,16,19,20)}$, and determining the approach to school lunch guidance by teachers, although not in the form of intervention research ${ }^{21)}$. There was also one paper describing research conducted for the purpose of accepting cultural differences relating to food culture $^{17)}$.

\section{Contents of food and nutrition education in} school lunches

The people in charge of food and nutrition education in the classroom shown in Table 2 were reported to be in the form of a team comprising a diet and nutrition teacher, homeroom teacher and subject instructor (three papers) ${ }^{13,15,18)}$, a single supervisor such as the homeroom teacher or subject instructor in two papers ${ }^{11,17)}$, and other people (three papers) ${ }^{12,14,16)}$. The contents of the classroom courses shown in Table 2 were classified based on curriculum guidelines. The numbers of papers found for each category consisted of two papers indicating home economics $^{11,13)}$, one paper indicating social studies in the area of course studies ${ }^{15)}$, no papers on moral education, one paper indicating foreign language activities ${ }^{17)}$, one paper indicating the period for integrated studies ${ }^{11)}$ and four papers indicating special activities ${ }^{12,14,16,18)}$.
Specific methods used to implement food and nutrition education for second graders included intervention through picture storytelling and panel theater performances ${ }^{14)}$. Methods targeted at fourth graders consisted largely of learning in the classroom, including experiments relating to the five senses and hands-on learning involving eating school lunch using the five senses, discussion on things noticed when eating ${ }^{20)}$, lectures utilizing 10 minutes before lunch ${ }^{19)}$ or learning using time allotted for social studies or integrated studies ${ }^{15}$. Methods targeted at fifth graders consisted of the posting of school lunch news announcements or distributing food and nutrition education news bulletins, as well as learning in the classroom using time allotted for home economics ${ }^{13)}$. During this time, the program involved learning about dietary balance as well as actually preparing properly balanced menus and lunches. There were numerous reports of classroom learning methods targeted at sixth graders and these included learning about food culture using time allotted for foreign language activities ${ }^{17)}$, using time allotted for home economics in the same manner as fifth graders ${ }^{13)}$, lectures using the 10 minutes prior to lunch ${ }^{19)}$, and learning related to staple foods, main dishes and side dishes through homeroom activities ${ }^{16)}$. Other reports described the posting of school lunch news announcements ${ }^{12)}$ and the distribution of food and nutrition education news bulletins ${ }^{13)}$. Methods targeted at first year junior high school students were reported to include the posting of school lunch news announcements ${ }^{12)}$.

According to reports describing methods targeted at all grades, activities included the holding of events allowing children to confirm school lunch leftovers and the manner in which they are cleaned $\mathrm{up}^{21)}$, the provision of menus featuring home-grown foods held once a month as part of a school-wide food and nutrition education program ${ }^{18)}$, the holding of school lunch gatherings combining different grades, and guidance on how to hold chopsticks and on the proper posture when eating held twice a month for the purpose of learning table manners. In the case of classroom learning this included the holding of food and nutrition education classes, and the creation of songs for the purpose of enhancing a sense of appreciation for food producers and preparation staff.

There was also one report targeted at parents instead of elementary school or junior high school students ${ }^{18)}$. This 


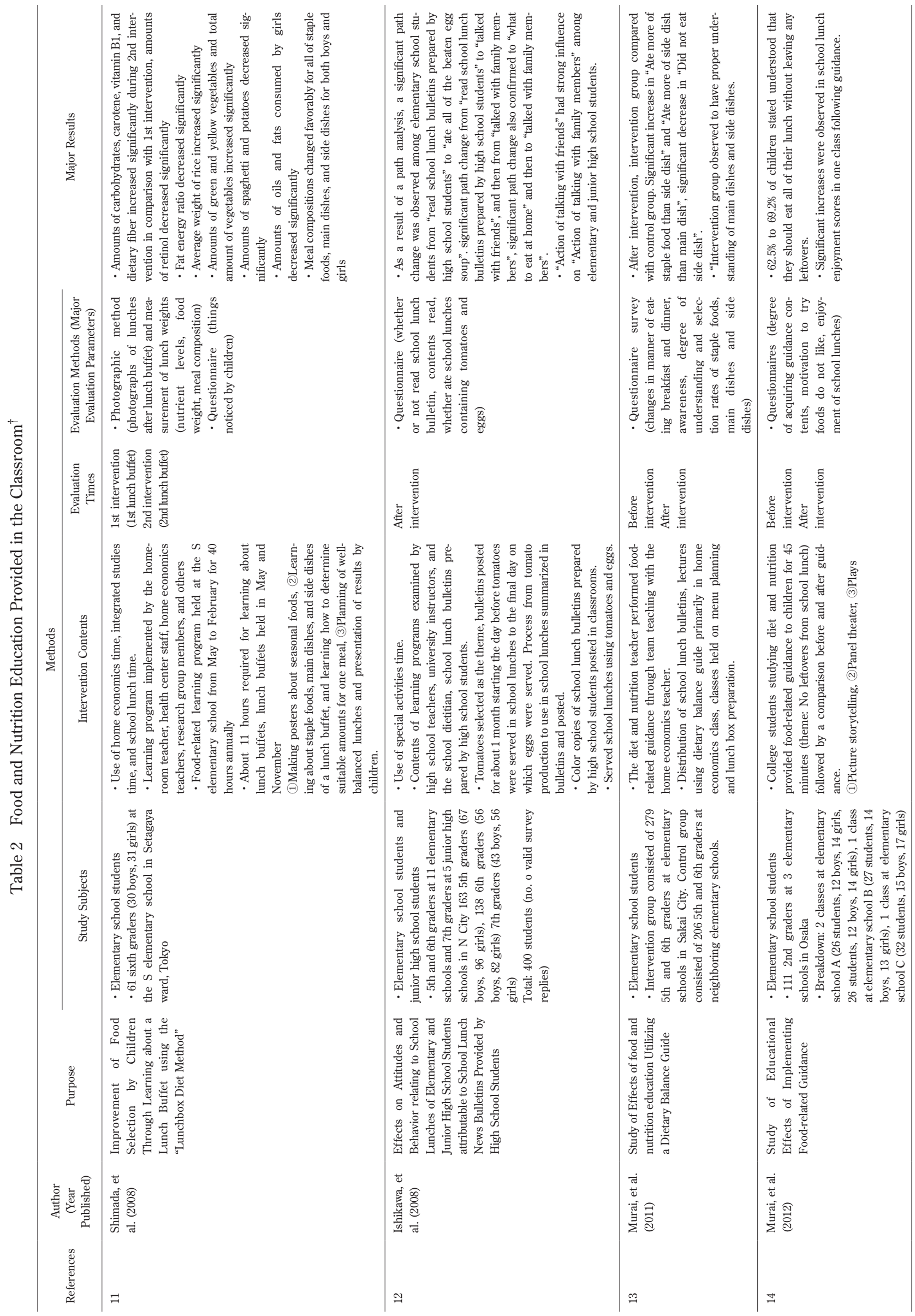




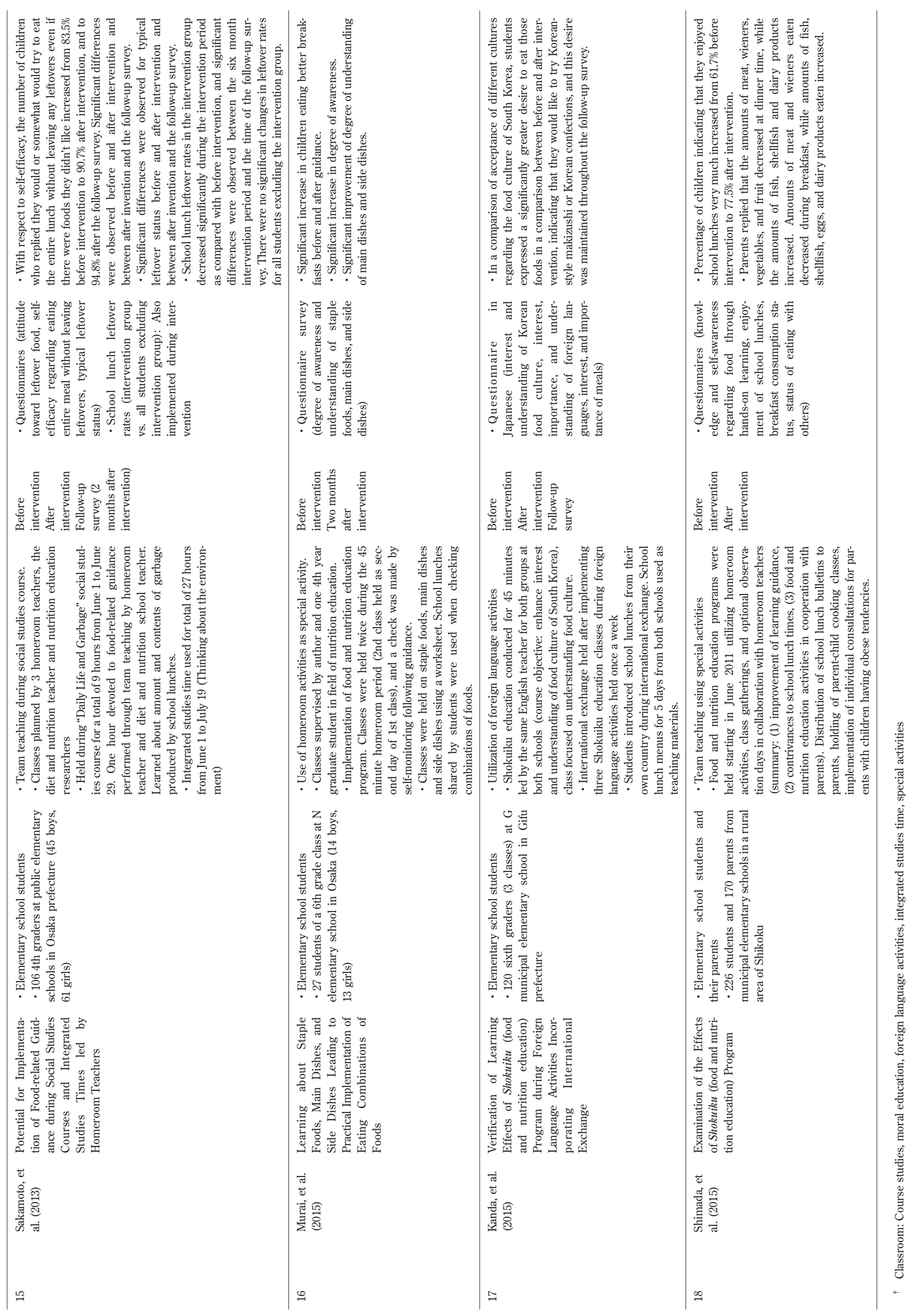




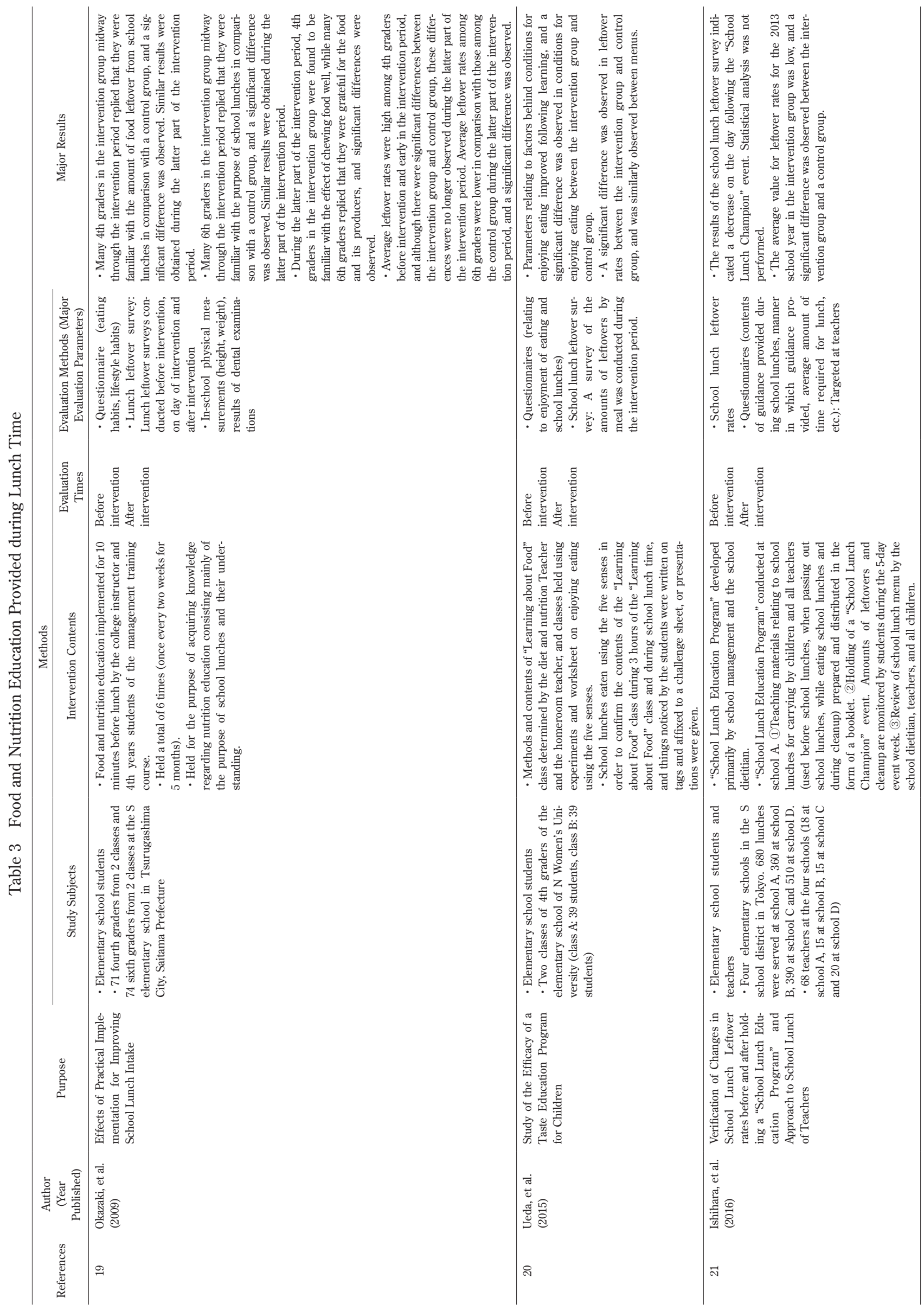


report described the distribution of school lunch news bulletins once a month and food and nutrition education news bulletins twice a month, individual consultations targeted at parents of children with obese tendencies, and the holding of lecture meetings and parent-child cooking courses focusing on the importance of good food and nutrition.

The contents of food and nutrition education were classified into six categories according to the "Food-Related Guidebook"23) published by the Ministry of Education, Culture, Sports, Science and Technology (MEXT) in March 2010. These categories included "the importance of food," "mental and physical health," "ability to select foods," "sense of gratitude," "social responsibility" and "food culture." The respective numbers of papers consisted of one paper on the "importance of food,"18) including guidance for its enjoyment and on regular eating; four papers on "mental and physical health"13, 14,16,19) including understanding the desirable way to consume nutrients and meals, reviewing one's own eating habits and attempting to form better eating habits; two papers on "ability to select foods"11,18) including the ability to acquire proper sound knowledge and make judgments relating to food; five papers on "sense of gratitude"12, 15, 19 21) such as not leaving food on the plate and appreciating those responsible for producing food; two papers on "social responsibility"15, 21) including the practical implementation of eating habits that give consideration to the environment and natural resources; and one paper on "food culture"17) targeted at enhancing understanding of Japanese culture and food culture.

6. Evaluation methods and outcomes of food and nutrition education at school lunches

Evaluations were performed using the questionnaire survey method in all the studies examined with seven papers $^{11,12,15,17,18 \sim 20)}$ determining food-related awareness; three papers ${ }^{13,14,16)}$ determining awareness and degree of understanding and a fact-finding survey of school lunches ${ }^{21)}$. In addition, dietary surveys were conducted in 10 papers, with five reports describing quantitative determinations ${ }^{11,15,19 \sim 21)}$ and five describing qualitative determinations ${ }^{12 \sim 14,16,18)}$. The quantitative survey methods used included photographic methods in one paper ${ }^{11)}$ and calculation of leftover rates in four papers ${ }^{15,19 \sim 21)}$. One paper also contained a report that included physical mea- surements and dental examination results in addition to a dietary survey ${ }^{20)}$.

\section{Discussion}

This study provides the first report of trends in food and nutrition education utilizing school lunches conducted in Japan. It consists of a systematic review relating to research verifying the effects of food and nutrition education in school lunches targeted at elementary school or junior high school students, the findings from which are reported below.

\section{Number of papers}

The systematic review focused on papers published after the enactment of the Shokuiku Basic Act in 2005. The papers that met the research criteria were published from 2008 following a revision of the School Lunch Act, with six of the total of 11 selected papers containing reports undertaken in the period 2015 2016, ten years after the enactment of the Shokuiku Basic Act. The number of reports relating to food-related guidance during school lunches has increased in recent years, and activities were suggested for implementation during school lunches as part of the educational curriculum. The papers selected comprised eight papers describing intervention contents related to food and nutrition education conducted in the classroom, and three papers describing intervention contents relating to food and nutrition education that utilized school lunch times.

Following legislation of the Diet and Nutrition School Teacher System and the revision of the School Lunch Act, reports have been published relating to the effects of food and nutrition education. This indicates that the role of the diet and nutrition school teacher is in line with the inherent objective of deploying food and nutrition education utilizing school lunches, and that school lunches are being implemented in the form of educational activities as a part of the curriculum. During the course of primary screening, 117 papers were excluded on the basis of not satisfying the selection criteria. In this study, some of the selection criteria were that papers had to be original articles, written concisely and be practical accounts. Proceedings, case reports and commentaries were excluded. Consequently, although there were a large number of reports ${ }^{2428)}$ in the form of bulletins and practical activities that were 
suitable for the theme of this study, including the development of a food and nutrition education program using school lunches ${ }^{29)}$ and the effect of using food and nutrition education luncheon mats during school lunches ${ }^{30)}$, these were excluded because they did not fit the criteria.

Also excluded were the Super Shokuiku School Projects initiated in $2014^{31)}$. Although the majority of their contents have not yet been reported, a listing of the types of programs implemented is available on the MEXT ${ }^{32)}$ website. More recently, the results of analyzing the contents of food education programs implemented in 69 examples of Super Shokuiku School Projects during the 2014 and 2015 school years have been reported as reference materials ${ }^{33)}$.

The School Lunch Act ${ }^{2)}$ places the responsibility on the diet and nutrition teacher to provide food and nutrition education utilizing school lunch programs. The duties performed by diet and nutrition teachers include the provision of food and nutrition education in three areas: during school lunch time, in the classroom, and guidance in the form of individual consultations for school children having health issues relating to food. There was however no report describing interventions in the form of individual consultations extracted for this study. Although the selected papers included reports of individual consultations targeted at parents of children with obese tenden$\operatorname{cies}^{21)}$, descriptions regarding the effects of this intervention were not available. However, a report was found relating to the provision of stepwise dietary guidance and the effects thereof targeted at high school students requiring special needs education ${ }^{34)}$. Although this paper was not selected since this study is targeted at elementary or junior high school students, according to the School Lunch Act ${ }^{35)}$, high schools providing special needs education operate according to the Criteria for Provision of School Lunches. Schools for special needs education are operated with greater flexibility while giving ample consideration to such factors as the health status of individual students, daily living status and actual conditions in the community $^{35)}$. Since dietary management for students at schools for special needs education is intimately related to eating habits at home or in the dormitory as well as meals served at hospitals - homeroom teachers, diet and nutrition teachers, school dietitians, school nurses, school physicians, primary physicians, parents and other related persons were shown to collaborate in an attempt to imple- ment dietary management that takes into view lifestyle habits of students while striving towards a common understanding. Individual consultation and guidance needs to be provided by the diet and nutrition teacher on a daily basis corresponding to the actual circumstances ${ }^{35)}$.

In its "Future School Lunch Program Centering on Diet and Nutrition Teacher"36) released in March 2017 MEXT indicates practical methods for individual consultations and guidance provided by diet and nutrition teachers. Examples provided of likely areas for individual guidance include "unbalanced eating tendencies," "obese tendencies," "under-eating tendencies," "food allergies" and "sports." Individual guidance is expected to be effective since it allows students to receive in-depth guidance and advice that takes advantage of the expertise offered by diet and nutrition teachers. In the future, there is expected to be an increase in the number of these practical case studies resulting in the development and sharing of practical educational practices and evaluation methods.

During the course of secondary screening which involved the careful reading of papers selected during primary screening, 34 papers described investigative research for dietary and nutritional management, accounting for a large number of the papers. In the report released by MEXT entitled, "Shokuiku henceforth centering on Diet and Nutrition Teachers"36), each of the programs promote dietary and nutritional management as an integral part of school lunch management by striving to achieve a correlation with annual guidance programs implemented in the classroom. Previous research reports that duties around school lunches account for a large part of dietary and nutritional management $t^{37)}$ and are required for proper and safe school lunches ${ }^{38)}$. There is therefore thought to be a large number of research papers relating to dietary and nutritional management. Although a large number of reports relating to investigative research and basic research ${ }^{39 \sim 66)}$ were identified, only 11 papers contained reports of interventions involving food and nutrition education, and coordination between basic research and practical research such as intervention research is recommended for the future.

\section{Purpose and contents of food and nutrition education during school lunches}

When the contents of food and nutrition education were examined using the classifications advocated by MEXT, 
the results indicated that the most frequently observed contents fell under the category of a "sense of gratitude," including placing importance on food and not leaving any leftovers as an expression of gratitude. However, this is thought to be due to a correlation between the methods used to evaluate food and nutrition education and outcome indicators. Since surveys on the amount of food left over can be conducted quantitatively facilitating comparative studies, they can also be used to assess the outcome of guidance. Among the 11 papers ultimately selected, leftover rates were calculated in four papers ${ }^{15,19 \sim 21)}$. In addition, a large number of reports were identified in which the guidance deployed fell under the category of "mental and physical health." Food and nutrition education utilizing school lunches has been implemented in Japan for the purpose of maintaining and promoting the mental and physical health of elementary and junior high school students while addressing various food-related health issues associated with children. Education utilizing school lunches has been deployed to instill behavioral change, and impart knowledge regarding proper combinations of foods and suitable dietary intake per meal. This provides an understanding of the desirable way to consume nutrients and meals, thereby enabling students to manage their diets on their own.

Most of the studies showed that persons in charge of food and nutrition education and of food-related interventions implemented in the classroom took the form of a team comprising a diet and nutrition teacher, homeroom teacher and subject instructor. Food and nutrition education utilizing school lunch time was indicated in three papers ${ }^{19 \sim 21)}$. School lunch programs were provided five times a week ${ }^{35)}$, and since this gives an opportunity for hands-on learning through the eating of school lunches, it allows for systematic and continuous guidance leading to expectations of behavioral changes. However, in the case of instruction provided only during school lunch times, it is difficult to verify and evaluate effectiveness due to the short time period, and this is thought to be responsible for the small number of intervention reports. In the future, guidance will be required that combines food and nutrition education in the classroom with school lunch times which provide an opportunity for hands-on learning on a daily basis. In addition, many of the papers targeted by this study contained ambiguous reports that were not system- atic or organized. Since food and nutrition education is implemented at schools to achieve education goals, it is considered important to publish reports demonstrating a clear understanding of correlation by clarifying the manner in which food and nutrition education is positioned.

3. Evaluation methods and outcomes of food and nutrition education during school lunches

According to the School Lunch Act, food and nutrition education utilizing the school lunch program is positioned as one of the duties of the diet and nutrition teacher. When considering that food and nutrition education is legally positioned in this manner, evaluation of educational effects is considered to be one of the responsibilities of the diet and nutrition teacher, and research reports in this area are expected in the future.

Ministry of Education, Culture, Sports, Science and Technology described the evaluation of food and nutrition education in its "Future School Lunch Program Centering on the Diet and Nutrition Teacher"36) released in March 2017. Although this report indicated knowledge relating to food, awareness of food, eating habits, lifestyle habits, obesity and under-eating as evaluation parameters of food and nutrition education, evaluation indices are not indicated for each parameter. The indices used to evaluate food and nutrition education as well as the methodology, are major issues. Discussions ${ }^{47)}$ and proposals ${ }^{48)}$ have been made relating to the evaluation of food and nutrition education at schools, and evaluation theories and techniques are likely to be devised. The evaluation methods revealed in this study included photographic methods that were examined for validity ${ }^{11)}$. In many of the other reports, questionnaire forms and worksheets were used that were proprietarily developed by the person providing guidance. In future, the establishment of evaluation theories and techniques and the development of questionnaire forms that have been examined for validity are expected to result in an increase in the number of studies using these tools along with facilitated comparisons with previous studies.

\section{Novelty and limitations of this study}

This study provided the first report of a systematic review of trends in food and nutrition education utilizing school lunches implemented in Japan and has identified the contents of food and nutrition education following legislation of the Diet and Nutrition Teacher system and 
revision of the School Lunch Act. The purpose of this study was to gather papers as comprehensively as possible, proceed with research using systematic review techniques, and combine the use of a database search and a manual search. Since six papers were selected on the basis of a manual search, it is thought that failure to gather important papers was minimized.

With respect to the limitations of this study, since the target papers were restricted to domestic journals and the database search was limited to the JMAS database, there is an issue with regards to the limited number of papers reviewed. Although the search format was carefully constructed, the existence of applicable papers that did not register a hit with the search words used in this study cannot be denied. The omission of papers from the search could therefore not be completely prevented. Although a manual search was conducted in conjunction with the database search for this reason, given that the search was limited to only certain journals, the study did not target all papers published in Japan, and there is the possibility that some studies were overlooked. Although decisions on which papers were selected were made after two persons carefully examined the papers and discussed their results, the presence of bias in making subjective judgments cannot be ruled out.

\section{Conclusion}

In this study, a systematic review of research papers was conducted to determine trends in food and nutrition education in Japan utilizing school lunches following the enactment of the Shokuiku Basic Act in 2005, the revision of the School Lunch Act in 2008 and legislation regarding the Diet and Nutrition Teacher system. A total of 11 papers were ultimately selected. These reports suggested that the role of a diet and nutrition teacher is in line with the inherent objective of deploying food and nutrition education utilizing school lunches, and that school lunches are being utilized as part of the educational curriculum. In particular, a large majority of food and nutrition education was observed to be taught in the classroom, and this was reported to lead to favorable changes. In the future, steps to promote behavioral changes are expected to be taken by implementing guidance using school lunch times, which serves as an opportunity for hands-on learning roughly 190 times a year, in combination with food and nutrition education provided in the classroom.

\section{Conflict of Interest}

This study does not contain matters constituting a conflict of interest.

\section{References}

1) Kanbe, M.: School lunch program and food and nutrition education, Current Information of Maternal \& Child Health, 65, 41-45 (2012) (in Japanese)

2) Matsukawa, N.: Revision of school lunch law, Jpn.J. School Health, 50, 337-339 (2008) (in Japanese)

3) Eto, K., Kishida, E., Kitabayashi, M., et al.: Recent trends of school-based nutrition interventions for children and adolescents in foreign countries- a systematic review, JJHEP, 19, 183-203 (2011) (in Japanese)

4) Nakatsu, T., Nagasaka, Y.: Systematic review of the studies of educational programs in the cooking classes from the view point of "Shokuiku", Archives of Yamaguchi Prefectural University, 9, 165-171 (2016) (in Japanese)

5) Makimoto, K. ed: Chapter 5, Systematic review of quantitative research, Systematic review for evidence-based nursing practice, pp. 61-74 (2013) Japan Nursing Association Publisher, Tokyo

6) Kunisato, Y.: Improving the methodological quality of systematic reviews and meta-analyses, Japanese Journal of Behavior Therapy, 41, 3-12 (2015) (in Japanese)

7) Kaneda, F., Sugano, S., Sano, A., et al.: Systematic review of the frequency of "Thinness" among children and adolescents in Japan, J. Nutr. Diet., 62, 347-360 (2004) (in Japanese)

8) Iwabe, M., Iwaoka, M., Yoshiike, N.: A systematic review of the effect of educational programs for promotion vegetable consumption among children in Japan, Jpn. J. Nutr. Diet., 72, 2-11 (2014) (in Japanese)

9) Japan medical abstracts society. "Ichu-Shi Web”: User's Manual Japan. (in Japanese). http://lib.tmd.ac.jp/seminar/Ichushi/ichushi_ver5_2012.pdf\#search=\%27\%E5\%8C\% BB\%E5\%AD\%A6\%E4\%B8\%AD\%E5\%A4\%AE\%E9\%9B\%91\%E8\% $\mathrm{AA} \% 8 \mathrm{C}+\% \mathrm{E} 8 \% 8 \mathrm{~B} \% \mathrm{~B} 1 \% \mathrm{E} 8 \% \mathrm{AA} \% 9 \mathrm{E} \% 27$ (Accessed September, 28, 2017)

10) Sugano, S., Kaneda, F., Yoshiike, N.: Points of use the Japanese literature database for research of nutrition science- a literature survey for the systematic review by using Japanese Medical Abstracts Society, J. Nutr. Diet., 61, 205-208 (2003) (in Japanese)

11) Shimada, M., Kobayashi, Y., Sakaguchi, A., et al.: Improvement of food choices among sixth-grade elementary school children before and after the nutrition education program using a buffet-style school lunch with the "Lunchbox Diet Method", Japanese Journal of Health Education and Promotion, 16, 94-109 (2008) (in Japanese) 
12) Ishikawa, M., Kubota, N., Okubo, M., et al.: Impact on attitude and behavior related to school lunch elementary pupils and junior high school students through school lunch newsletter prepared by agricultural high school students, Journal of the Japan Dietetic Association, 51, 26-36 (2008) (in Japanese)

13) Murai, Y., Yatsutake, M., Okuda, T.: The effect of dietary education using the Japanese food dietary balance guide spinning top among elementary school children, Journal of Japanese Society of Shokuiku, 5, 9-18 (2011) (in Japanese)

14) Murai, Y., Maruya, N., Yamamoto, R., et al.: The effects of university student-taught dietary education by students for the purpose of food and nutrition education: practical for second graders, Journal of Japanese Society of Shokuiku, 6, 173-181 (2012) (in Japanese)

15) Sakamoto, T., Yachiku, M., Haruki, T.: Study on the feasibility and learning outcomes of nutrition education in social studies and integrated studies that led primarily by the homeroom teacher: aiming to form the behavior of eating school lunch without food waste, Japanese Journal of Nutrition and Dietetics, 71, 76-86 (2013) (in Japanese)

16) Murai, Y., Ando, H., Yamazaki, N., et al.: Effects of a dietary education program to eat a combination of staple foods, main dishes and side dishes: focusing on breakfast guidance for 6th targeted at elementary school students, Journal of Japanese Society of Shokuiku, 9, 105-112 (2015) (in Japanese)

17) Kanda, S., Imai, A., Fujikura, J., et al.: The practical of shokuiku (eathig education) through an with Korea in "foreign language activities" classes in Japanese elementary scool, Journal of Japanese Society of Shokuiku, 9, 81-91 (2015) (in Japanese)

18) Shimada, S., Wakabayashi, Y., Okamura, K., et al.: The practice and effects of shokuiku program with nutrition teachers at its core: passed on the case of elementary school, Journal of Japanese Society of Shokuiku, 9, 27-39 (2015) (in Japanese)

19) Okazaki, M., Iso, N., Mitsuhashi, T.: Practical and the effects of nutrition education program to increase the among of children's intake of the school lunch, Journal of Japanese Society of Shokuiku, 3, 49-64 (2009) (in Japanese)

20) Ueda, Y., Ohtawara, M., Nagai, A.: Implementation of education on fourth graders and its effects evaluation, Journal of Japanese Society of Shokuiku, 9, 247-255 (2015) (in Japanese)

21) Ishihara, Y., Ohkawa, M., Takamasu, M.: Use of a "School lunch study program" in elementary school lunch guidance, Journal of Japanese Society of Shokuiku, 10, 203-213 (2016) (in Japanese)

22) Ministry of Education, Culture, Sports, Science and Technology, Japan: The Course of Study. (in Japanese). http://www.mext.go.jp/a_menu/shotou/new-cs/ youryou/syo/index.htm (Accessed September, 28, 2017)

23) Ministry of Education, Culture, Sports, Science and Technology, Japan: Food-Related Guidance Guidebook.
(2014). (in Japanese). http://www.mext.go.jp/a_menu/ sports/syokuiku/1292952.htm (Accessed September, 28, 2017)

24) Ishihara, Y.: Establishment of "Food and nutrition education day" towards Improvement of breakfast intake rate, Journal of the Japan Dietetic Association, 59, 546-547 (2016) (in Japanese)

25) Kamakura, M.: Establishment of desirable eating habits - from a class of elementary school third graders, Journal of the Japan Dietetic Association, 59, 174-175 (2016) (in Japanese)

26) Fujiki, Y.: Food-related guidance at special needs schools - learning how to eat more safely, Journal of the Japan Dietetic Association, 58, 877 (2015) (in Japanese)

27) Sugi, H.: Raising children for developing healthy bodies, Journal of the Japan Dietetic Association, 56, 553 (2013) (in Japanese)

28) Koike, Y.: What school lunch program can do for lifelong health maintenance - contrivances to food and nutrition education activities using local foods, Journal of the Japan Dietetic Association, 56, 623 (2013) (in Japanese)

29) Kurahashi, N., Wakabayashi, K., Hayakawa, M., et al.: Development of a dietary education program using school lunches and experiential activity - study in one elementary school in inuyama city, Journal of Nutrition and Medical Science, 1, 21-28 (2012) (in Japanese)

30) Suzuki, Y., Sakaguchi, M., Tanaka, S., et al.: Effect of use of the developed "Food and nutrition education luncheon mat" in school lunch time in elementary school, Bulletin of the Center for Educational Research and Teacher Development, 19, 223-227 (2010) (in Japanese)

31) Ministry of Education, Culture, Sports, Science and Technology, Japan: The super shokuiku school projects. (in Japanese). http://www.mext.go.jp/b_menu/houdou/ 26/04/__icsFiles/afieldfile/2014/04/11/1346607_01_1. pdf (Accessed January, 15, 2018)

32) Ministry of Education, Culture, Sports, Science and Technology, Japan: About the contents of the super shokuiku school projects. (in Japanese). http://www. mext.go.jp/a_menu/sports/syokuiku/1353381.htm (Accessed January, 15, 2018)

33) Hijikata, N., Nakaoka, K., Goseki-Sone, M., et al.: Application of results from the super shokuiku school projects, Japanese Journal of Nutrition and Dietetic, 75, 164-173 (2017) (in Japanese)

34) Maeda, N.: Training of eating skills for the two intellectually retarded youth autism, The Japanese Journal Autistic Spectrum: Practice and Clinical Reports, 10, 73-80 (2013) (in Japanese)

35) Ministry of Education, Culture, Sports, Science and Technology, Japan: Criteria for Provision of School Lunches. (in Japanese). http://www.mext.go.jp/b_menu/ hakusho/nc/k19540928001/k19540928001.html (Accessed January, 15, 2018)

36) Ministry of Education, Culture, Sports, Science and Technology, Japan: Shokuiku henceforth on centering on 
the Diet and Nutrition Teachers - PDCA of Shokuiku promotion grappling with team schools. (2017). (in Japanese). http://www.mext.go.jp/a_menu/sports/syokuiku/1385 699.htm (Accessed January, 15, 2018)

37) Toda, M., Homma, T.: Present state of dietary education by school dietitians - Focusing on nationwide elementary and junior high school, Journal for the Integrated Study of Dietary Habits, 15, 194-201 (2004) (in Japanese)

38) Hosoyamada, Y., Kosuge, M., Toyomitsu, M.: Action of dietitians regarding school lunch program based on newspaper articles; investigating the number of newspaper before and after revision of the School Lunch Act, Journal of the Japan Dietetic Association, 58, 41-47 (2015) (in Japanese)

39) Nozue, M., Kyungyul, J., Ishihara, Y., et al.: Difference in food consumption and distribution of meals between the days with or without school lunches among $5^{\mathrm{TH}}$ grade elementary school students, Japanese Journal of Nutrition and Dietetics, 68, 298-308 (2010) (in Japanese)

40) Hayashi, N., Imai, T., Hasegawa, M., et al.: Comparative study of quality of life (QOL) of eating habits of children with and without food allergies, Japanese Society of Pediatric Allergy and Clinical Immunology (0914-2649), 23, 643-650 (2009) (in Japanese)

41) Nakanishi, A., Okubo, H., Takamura, M., et al.: Number of servings per dish eaten by $5^{\text {th }}$ grade children - Implications for better use of the Japanese food guide spinning top among school children, Japanese Journal of Nutrition and Dietetics, 67, 128-140 (2009) (in Japanese)

42) Suga, A., Suzuki, S., Tobiyama, Y., et al.: Effects on food awareness and lifestyle habits of school children of employment and working hours of mothers, Japanese Journal of Sports Nutrition, 5, 43-49 (2012) (in Japanese)

43) Abe, K., Akamatsu, R.: Factors contributing to food waste during lunchtime in elementary schools, Japanese Journal of Nutrition and Dietetics, 69, 275-281 (2011) (in Japanese)

44) Kojima, Y., Abe, A., Abe, K., et al.: The relationship between food waste from school lunches and nutrient intake in elementary school children, Japanese Journal of Nutrition and Dietetics, 71, 86-93 (2013) (in Japanese)

45) Toyama, M., Abe, K., Akamatsu, R.: Leftover-related behaviors and cognition, and instruction to eat everything served at home among Junior high school lunch program, Japanese Journal of Nutrition and Dietetics, 71, 350-356 (2013) (in Japanese)

46) Toyama, M., Fukuoka, K., Akamatsu, R.: Factors contributing to food waste during lunchtime in junior high schools, Japanese Journal of Health Education and Promotion, 23, 35-42 (2015) (in Japanese)

47) Akamatsu, R.: Towards a discussion of "Evaluation of food and nutrition education at school”, Japanese Journal of Health Education and Promotion, 22, 247-248 (2014) (in Japanese)

48) Akamatsu, R., Inayama, T., Gotoh, K., et al.: Evaluation of food and nutrition education at school aimed at formation of desirable eating habits, Japanese Journal of Health Education and Promotion, 23, 145-151 (2015) (in Japanese)

(Received September 29, 2017; Accepted June 5, 2018) 